\title{
In search of referents for nouns and pronouns
}

\author{
HERBERT H. CLARK and C. J. SENGUL \\ Stanford University, Stanford, California 94305
}

\begin{abstract}
In comprehending a sentence, people normally try to identify the objects, events, and states referred to by its definite noun phrases. In Experiment 1, subjects took less time comprehending a sentence when the referents of such noun phrases were mentioned one sentence back than when they were mentioned two or three sentences back. There appeared to be a discontinuity between the first and second sentences back. Experiments 2 and 3 showed that it was most likely the first clause back, not the first sentence back, that was critical. These findings suggest that, independent of other factors, the last clause processed grants the entities it mentions a privileged place in working memory. They are readily available to be referred to by nouns and pronouns.
\end{abstract}

When we hear "she" in an utterance, we normally feel compelled to identify the entity to which it refers before we feel we have understood the utterance fully. The speaker, we assume, had some particular female in mind and expected us to identify her uniquely from what we know at that point in the conversation. Often, this means searching memory for a female mentioned in the last sentence or two, but at other times it requires a more complicated search and inference (Springston, 1975; Charniak, Note 1). And what is true for definite pronouns like "she" is also true for definite noun phrases like "the woman." The present study is an investigation of the process by which listeners search memory for such referents in the natural course of comprehension.

How listeners identify referents is part of the broader question of how listeners extract the novel information conveyed by an utterance and incorporate this information into memory. According to one model (Clark \& Haviland, 1974, 1977; Haviland \& Clark, 1974), this is accomplished in part by the given-new strategy. In individual sentences, there are structural properties that distinguish between given information, that portion of the sentence that is supposed to refer to information the speaker believes his listeners can identify, and new information, that portion that is supposed to contain information the speaker believes his listeners do not yet know. In the given-new strategy, listeners add the new information of a statement to memory in three steps. First, they identify the given and new information of the sentence from its structural cues. Second, they search memory for a unique referent of the given information-that entity (object, state, event, or process) to which the given information refers. If they find no such referent directly, they add, based on what they

The research reported here was supported in part by Grant MH-20021 from the National Institute of Mental Health. We thank Eve V. Clark for comments on earlier drafts of the paper. already do know, a plausible bridging assumption that contains the referent. Third, they add the new information to memory by attaching it to the referent they have just identified or added. A good deal of evidence for this strategy has been laid out by Clark and Haviland (1977; see also Carpenter \& Just, 1978; Clark, 1977, 1978; Hupet \& LeBouedec, 1977; Yekovich \& Walker, 1978).

How does the given-new strategy apply to definite noun phrases and pronouns? When listeners encounter "the woman" or "she," they are assumed to treat this as given information for which they must find a referent. They then search memory for the unique entity to which "the woman" or "she" was intended to refer. When they identify that referent, they attach to it the new information being conveyed about "the woman" or "she." In those cases where there is already in memory a unique entity to which "the woman" or "she" refers, will listeners ever have difficulty searching for and identifying it? Clearly, they will, and for a variety of different reasons. In this paper, we will examine just one of those reasons, the relative availability of the referent in working memory.

The availability of a referent in memory ought to depend on where and how it was mentioned in the prior discourse. (We will not consider cases in which the referent has not been explicitly mentioned in the prior discourse; see Clark, 1977.) Consider this sequence of three sentences: (1) Yesterday I met a woman who had written a book on viruses. (2) She had studied them for years and years. (3) It was selling very well. The pronoun "she" in Sentence 2 refers to the woman mentioned in Sentence 1; its referent seems easy to identify. The pronoun "it" in Sentence 3 refers to the book mentioned in Sentence 1; its referent seems more difficult to identify. What is the difference? Informally, it looks as if a referent is readily identifiable if it was mentioned one sentence back. It seems less available for identification if it was mentioned two sentences back. Notice how much easier "it" becomes when 
Sentence 3 comes right after Sentence 1 , so that its referent is mentioned just one sentence back.

If what is critical here is merely when in the prior discourse the referent was mentioned last, there are two obvious models of the search process. In the "continuity model," the entities mentioned in a discourse are laid down in memory like beads on a string. The entities are strung one by one as they are mentioned in the discourse. Their order on the string mirrors their order of mention in the discourse. When listeners try to identify the referent of a noun or pronoun, they search these beads from the final one backward. The further back they have to search, the longer they should take and the slower they should be in understanding the present sentence.

The "discontinuity model" is like the continuity model except that it has two strings of beads instead of one. The first, and privileged, string contains the entities mentioned in the current sentence and one sentence back, while the second string contains the entities mentioned two or more sentences back. The entities for the first sentence back are transferred to the second string when the current sentence is completed, and so all that remains on the first string are the entities from the just-completed sentence. The premise of this model is that the first string, the entities mentioned in the current sentence and one sentence back, has a privileged place in working memory and so is readily available for examination, for search and identification. The second string is not in working memory and takes extra time and effort to examine. Part of this string may even lose its identifiability. As the name suggests, therefore, the discontinuity model predicts a discontinuity in the search process. Referents will be identified readily only if they are mentioned in the current sentence or one sentence back. Several experiments reported by Carpenter and Just (1978) provide considerable evidence that the farther back a referent is mentioned, the more difficult it is to identify. But these findings do not distinguish clearly between the continuity and discontinuity models, although they suggest a discontinuity one sentence back.

Why might one expect such a discontinuity? The answer lies in the function of definite noun phrases and pronouns in making discourse coherent. Discourse generally has a thematic continuity. Topics tend to be taken up one at a time, with each new sentence adding further information to the topic that has been introduced. As Grice (1975) put it, each sentence is expected to be relevant. Definite noun phrases are special devices for showing how the current sentence is relevant, for they bind the facts now being put forward to entities that have already been mentioned. But there must be a limit on how remotely this binding can occur, because listeners, with their limited working memories, cannot possibly keep all the entities connected with a topic in mind at once. The general compromise may be for listeners to give the entities mentioned in the last sentence a privileged status in working memory, since those are the entities the speaker is most likely to refer to.

In their strong forms, however, both models are wrong on the face of it. The entities mentioned in a discourse are surely arranged not in a string, a linear ordering of entities, but in some sort of hierarchical structure. Consider this sequence: (4) Max managed to meet Rudolph at the party. (5) Then he left. Even though Rudolph is mentioned more recently than Max, "he" in Sentence 5 is normally taken to refer to Max. This and many other examples demonstrate that what a pronoun is taken to refer to depends crucially on the structure of both the sentence containing the pronoun and the sentence mentioning its referent (see, e.g., Caramazza, Grober, Garvey, \& Yates, 1977; Garvey, Caramazza, \& Yates, 1976). Nevertheless, there may still be a continuity or discontinuity in availability between the entities mentioned one sentence back and those mentioned two or more sentences back. It is in this weaker sense that we intend the continuity and discontinuity models to be construed.

For thematic reasons, other entities may be granted a privileged status too. Chafe (1974) has argued that speakers generally design given information to refer to entities they believe their listeners have "in consciousness" at the time of utterance. Such entities will almost always include those mentioned in the last sentence, but they will also include such things as the topic of the discourse, the scene, and other generally assumed facts, like the identities of the speaker and listeners. All of these will be "on stage" and ready to be referred to. In the present study, we were interested in isolating the possible privileged status of the last sentence in working memory, and so we have systematically excluded from consideration entities concerned with the topic, scene, or generally assumed facts. Our experiments have little to say about the possible privileged status of any of these other entities.

The privileged status of the entities mentioned one sentence back is not the same thing as the verbatim memory for that sentence. Jarvella (1971) has shown that, while the current sentence is readily available for verbatim recall, the sentence one back is not. There is a sharp drop in verbatim backward recall of a sentence once the listener is into the following sentence. So, if the discontinuity model is supported, that will suggest that searching working memory for a referent is not the same thing as searching memory for the particular word or phrase that referred to that referent. As most investigators have assumed, memory for the information conveyed by a sentence should be distinct from memory for its verbatim contents.

The present study was designed to investigate three issues. First, do the entities mentioned one sentence 
back have a privileged status in memory, as the discontinuity model claims? Second, if there is a discontinuity, does it occur at sentence or clause boundaries? And third, is the search for referents the same for pronouns as for nouns? There is some reason to think that pronouns, a particularly barren type of noun phrase, may initiate a type of search that places greater demands on the availability of referents (Chafe, 1974). There are also well known constraints on the relation of pronouns and their referents when both occur in the same sentence (see Jackendoff, 1972; Langacker, 1969; Less \& Klima, 1963; Postal, 1971; Ross, 1967), and Springston (1975) has shown that these constraints affect the search for a pronoun's referent. These intrasentential constraints could conceivably affect the search for referents across sentence boundaries too. But if the search is determined mainly by the status of the referents in working memory, the search should be the same for pronouns as for nouns.

\section{EXPERIMENT 1}

\section{Method}

On each of a series of trials, the subject (1) read a threesentence context paragraph presented in a tachistoscope, (2) pressed a button when he had completed it, (3) read a target sentence presented immediately afterward, and (4) pressed a button when he felt he understood that. The target sentence contained either a noun or pronoun whose referent had been mentioned in Sentence 1,2 , or 3 of the context paragraph. If the entities mentioned in Sentence 3 have a privileged status in working memory, then comprehension of the target sentence should be fast when the referent is mentioned in Sentence 3, but slow when it is mentioned in Sentence 1 or 2 . Note that comprehension time is subjective; it is how long the subject takes before he "feels he has understood the sentence." This measure has been used successfully before by Haviland and Clark (1974), and by all others who have used "reading time" as a measure of comprehension speed.

Each subject went through 10 practice trials and 96 experimental trials. The 96 experimental trials consisted of 16 trials of each of six types: The referent was mentioned in Sentence 1, 2 , or 3 of the context paragraph, and the target sentence contained either a noun or a pronoun. We composed all contexttarget sequences ourselves, basing them on sentences culled from short stories and novels on our bookshelves. The context paragraph always read like a simple description in which the three sentences had no intrinsic order. The paragraph mentioned the referent to the target nominal only once and made no other implicit reference to it. In all paragraphs, there were mentions of things that could serve as referents to other nouns or pronouns, so the subject could not readily guess which one would be referred to later. The mention of the referent was always indefinite. Here is one example of a context-target sequence; the referent in Sentence 3 is in italics: (Context) A broadloom rug in rose and purple colors covered the floor. Dim light from a small brass lamp cast shadows on the walls. In one corner of the room was an upholstered chair. (Target) The chair appeared to be an antique.

We constructed three counterbalanced lists of these 96 context-target sequences. There was a master list that had the 96 sequences, 16 of each type, in a random order. Lists 2 and 3 were constructed from the master list by retaining the 96 items in the same order, but by permuting the three context sentences for 72 of the sequences (12 of each type) in a Latin square design. Consider a context paragraph in the master list whose sentence order is $\mathrm{RXY}$, where $\mathrm{R}$ denotes the sentence mentioning the referent. Then, for this context paragraph, List 2 had the order XRY, and List 3, XYR. In brief, for each target sentence, one list mentioned the referent in context Sentence 1, another in Sentence 2, and a third in Sentence 3, all in a counterbalanced design. Sentences 1,2 , and 3 averaged 13.3 words (range 5-32), 12.7 words (range 5-32), and 12.1 words (range 5-21), respectively, and the target sentence averaged 6.6 words (range $5-10)$. The 24 context-target sequences that were identical in all three lists, 4 of each type, were of similar lengths; they will be called fillers and will be treated separately.

The context-target sequences were presented in a modified Iconix tachistoscope with a $13 \times 15 \mathrm{~cm}$ field split into two separate fields, an upper field and a lower field, each $7 \times 13 \mathrm{~cm}$. The context paragraph appeared in the upper field, and the target in the lower field, both typed in elite type on a white background. The displays were viewed at a distance of $51 \mathrm{~cm}$.

To begin each trial, the subject, after a signal from the experimenter, pressed a button, and, $.5 \mathrm{sec}$ later, the context paragraph appeared in the upper field. When the subject finished reading it, he pressed the same button again, at which time the context paragraph disappeared from the upper field and the target sentence appeared in the lower field. As soon as he felt he understood the target sentence, the subject pressed a second button with his other hand. The experimenter recorded the time in milliseconds from the appearance of the target sentence to the press of the second button. Between trials, there was a blank $13 \times 15 \mathrm{~cm}$ field that covered the area of the upper and lower display fields.

The subjects in this and the remaining experiments were Stanford University undergraduates, participating for credit in introductory psychology or for $\$ 2$ in pay. There were 24 subjects in this experiment, 13 men and 11 women, 8 receiving each of the lists. Two subjects were replaced, one for making more than $10 \%$ errors and another for not following instructions. Each subject was told he would be reading simple stories or descriptions, which he was to read at normal speed without studying or memorizing the material. The subject was instructed to press the button for the target sentence as soon as he understood it "as it fit in with the rest of the story." If he was unable to connect it to the preceding paragraph, he was to report this to the experimenter. These responses were recorded as errors, and so were presses of the wrong button. Each session lasted about $40 \mathrm{~min}$, with a short rest halfway through.

Method of analysis. The analysis of the latencies required three steps. The first step was to replace wild latencies. We began by eliminating the $1.9 \%$ error latencies and all latencies over $10 \mathrm{sec}$, and then all latencies more than 2.5 SDs from the mean of the six conditions for each subject. Finally, we replaced all missing scores, $4.4 \%$ of the total, by the procedure recommended by Winer $(1971$, p. 487). The means listed in Table 1 are drawn from the results of this first step.

The second step was to normalize each subject's latencies by use of the filler items. Since the 24 filler items were the same for all subjects, the mean latencies on this set should be comparable from subject to subject. We therefore computed the deviation of each subject's mean for the filler items from the overall mean for the filler items and corrected all of that subject's scores by that amount. In effect, we adjusted each subject's latencies so that all subjects would have the same mean latency on the filler items. This procedure was intended to reduce the latency variation confounding the subjects, their groups, and the conditions. This adjustment, of course, leaves the mean latencies listed in Table 1 unaltered.

The third and final step was to carry out an analysis of variance on the adjusted latencies. In accordance with recommendations of Clark (1973), we treated both items and subjects as random effects and computed the statistic min $F^{\prime}$. 
Table 1

Mean Comprehension Latencies for Target Sentences With Referents in One of Three Context Sentences

\begin{tabular}{lccc}
\hline & \multicolumn{3}{c}{ Context Sentence Mentioning Referent } \\
\cline { 2 - 4 } Nominal & 1 & 2 & 3 \\
\hline Nouns & 2174 & 2166 & 1802 \\
Pronouns & 2280 & 2133 & 1847 \\
Means & 2227 & 2150 & 1825 \\
\hline
\end{tabular}

\section{Results}

The mean latencies for the six conditions are listed in Table 1. They show that the target sentence was comprehended quickly when the referent was mentioned in Sentence 3 of the context paragraph, but equally slowly when it was mentioned in Sentence 1 or 2 . The referent was identified an average of $364 \mathrm{msec}$ faster in Sentence 3 than in Sentence 1 or $2\left[\min F^{\prime}(1,67)=18.99\right.$, $\mathrm{p}<.001$ ], whereas it was identified only $77 \mathrm{msec}$ faster in Sentence 2 than in Sentence $1\left(\min F^{\prime}<1\right)$. The 364$\mathrm{msec}$ figure is reliably greater than the $77-\mathrm{msec}$ figure $\left[\min \mathrm{F}^{\prime}(1,67)=5.06, \quad \mathrm{p}<.05\right]$. The nouns and pronouns were alike, with no reliable differences in their means or interactions $\left(\min \mathrm{F}^{\prime}<1\right.$ ). The main finding, then, is that there is a clear discontinuity in the latencies. The target sentence becomes distinctly harder to understand when the referent is more than one sentence back.

In subsidiary analyses of the mean latencies of the 72 target sentences in the three lists, we found little of interest. Comprehension time was expected to increase with the length of the target sentences in words, and it did $(r=.31, p<.005)$. But it did not increase the farther back from the target sentence the referent in Sentence 1,2 , or 3 alone was mentioned. The appropriate partial correlation was not reliable; number of words back was relatively unimportant once the number of sentences back had been accounted for. This is expected on independent grounds. As argued in relation to Sentences 4 and 5 in the introduction, it is often most natural to refer to the subject of a sentence even though the object of the verb or some other noun phrase is more recent. As Caramazza et al. (1977) and Garvey et al. (1976) have shown, what is taken to be the referent of a pronoun is generally governed by factors other than distance back within the sentence. Comprehension time, however, did correlate $(.32, p<.005)$ with the word length of the context paragraphs, which varied from 23 to 63 words, with a mean of 38 words.

\section{Discussion}

Experiment 1 provides a good case for the discontinuity model. Subjects identified the referent of a noun or pronoun much faster when it was mentioned in Sentence 3 than when it was mentioned in Sentence 2. They were only slightly faster when it was mentioned in
Sentence 2 than when it was mentioned in Sentence 1. They appeared to treat the referents of the nouns and pronouns in the same way. Discontinuities, of course, are a matter of degree, but here, the ratio of the difference between Sentences 2 and 3 to the difference between Sentences 1 and 2 was more than $4: 1$, which is substantial $\left[\min t^{\prime}(1,67)=1.49, p<.07\right]$. Subjects, furthermore, did not get any faster the later the referent was mentioned in Sentence 1,2 , or 3 taken alone, although this experiment was not designed to detect such differences with any sensitivity. In short, subjects found it easy to identify a referent as long as it was mentioned anywhere in Sentence 3, and they found it relatively hard otherwise.

But what kind of discontinuity is this? Experiment 1 suggests that the discontinuity occurs at sentence boundaries, but it may occur at clause boundaries instead. One way to distinguish these two possibilities is to manipulate the clauses and sentences of the context paragraph independently (see Jarvella, 1971). This was the purpose of Experiments 2 and 3.

\section{EXPERIMENT 2}

\section{Method}

The subjects in Experiment 2 carried out the same task as those in Experiment 1. The only changes were in the number and composition of the context-target sequences. And, unlike Experiment 1 , the critical nominals used here in the target sentences were definite noun phrases excluding pronouns.

Here, each subject received 10 practice and 80 experimental context-target sequences. Each context paragraph this time consisted of three clauses that were put together to form two sentences. One of the three clauses was a subordinate clause attached either before or after one of the other two main clauses. The four types of context paragraphs can be represented as follows: Type 1, sA, B. C; Type 2, A sB. C; Type 3, A. sB, C; Type 4 , A. B sC. Here, A, B, and C represent the three clauses, and $s$ represents one of the subordinate conjunctions "because," "while," "when," "after," "before," "although," "since," "as," "just as," "as soon as," and "in order to." In Types 1 and 2, note that B is part of the first sentence (Sentence 1); in Types 3 and 4 , it is part of the second sentence (Sentence 2). The basic question was whether a referent in Clause B would be easier to reach when it was part of Sentence 2 (as in Types 3 and 4) than when it was part of Sentence 1 (as in Types 1 and 2). Clauses A, B, and $C$ averaged 11.5 words (range 5-17), 11.0 words (range 6-17), and 11.1 words (range 5-19), respectively, and the target sentence averaged 7.0 words (range 5-9).

of the 80 context-target sequences, 40 mentioned the referent in context Clause B, 20 in $\mathrm{A}$, and 20 in C. For all three groups of items, there were equal numbers of Type 1, 2, 3, and 4 context paragraphs. The 80 sequences were placed in a random order to make up the master list. The 40 items with referents in Clause B were systematically altered to form a counterbalanced List 2, while the 40 items with referents in Clause A or C remained unchanged from List 1 to List 2 and were treated as filler items and analyzed separately. For the 40 items with referents in Clause $B$, there were changes of five items each of Type 1 to Type 3,1 to 4,2 to 3,2 to 4,3 to 1,3 to 2,4 to 1 , and 4 to 2 . Each change consisted of alterations in the punctuation, in the place, and sometimes in the type of subordinate conjunction, but in nothing else. The whole paragraph made just as much sense after the change as before. Here is an example 
of a change from Type 1 to Type 4: Type 1 (Context) Before the campers erected a shelter of lightweight plastic in the clearing, they piled a supply of dry wood next to a tree. The smell of coffee mingled with smoke from the campfire. (Target) The tree was an enormous redwood. Type 4 (Context) The campers erected a shelter of lightweight plastic in the clearing. They piled a supply of dry wood next to a tree while the smell of coffee mingled with smoke from the campfire. (Target) The tree was an enormous redwood. So each target sentence with a referent in Clause B appeared with Clause B in Sentence 1 in one list and with Clause B in Sentence 2 in the other list. The referent was the same number of words back (except sometimes for the added subordinate conjunction) in both contexts.

In all other respects, Experiment 2 was like Experiment 1. There were 24 subjects, 15 men and 9 women, 4 of whom were replaced for making more than $10 \%$ errors or for not following instructions. Here again, we went through the three-step analysis of the latencies, this time replacing $3.0 \%$ of the scores after using a 3.0-SD cutoff.

\section{Results and Discussion}

The mean latencies for the four types of context paragraphs are listed in Table 2. As they demonstrate, subjects were only $42 \mathrm{msec}$ faster when Clause B was part of Sentence 2 than when it was part of Sentence 1 . This difference was not reliable $\left[\min \mathrm{F}^{\prime}(1,55)=1.25\right]$, nor were any other main effects or interactions. This experiment failed to show any reliable effects at all.

So Clause B was not granted a privileged status in working memory when it was made part of the last sentence. The gain in comprehension time was only $42 \mathrm{msec}$, which is small compared to the $325-\mathrm{msec}$ advantage of Sentence 3 over Sentence 2 in Experiment 1 . Moreover, Clauses $\mathrm{A}, \mathrm{B}$, and $\mathrm{C}$ were about the same length as Sentences 1,2 , and 3 , so the two experiments were comparable in this respect. The findings suggest that it may not be the last sentence but the last major clause that is granted the privileged status in working memory. This notion was tested in Experiment 3.

\section{EXPERIMENT 3}

\section{Method \\ Experiment 3 was like Experiment 2 except that the context paragraphs were manipulated according to a slightly different design. Here, we were interested in whether the referent was in Clause $\mathrm{B}$ or $\mathrm{C}$, where either $\mathrm{B}$ or $\mathrm{C}$ was a subordinate clause. \\ Each subject received 10 practice and 96 experimental context-target sequences, all of Types 3 and 4 of Experiment 2. That is, all context paragraphs were either "A. sB, C" or "A. B sC." Of the 96 items, 24 mentioned the referent in Clause A, 36 in Clause B, and 36 in Clause C. These 96 items were placed in a random order to form the master list. List 2 was constructed by systematically altering all items with the referent in Clause B to ones with the referent in Clause $C$, and vice versa. The 24 items with the referent in Clause $A$ were the same for both lists and were considered fillers and treated separately. The change of each nonfiller context paragraph was brought about by reversing the two clauses in the second sentence without changing the subordinate conjunction. So if $B$ and $\mathrm{C}$ were in the relation " $\mathrm{X} \mathrm{SY}$ " on one list, they were in the relation " $\mathrm{Y} Y, X$ " on the other list, and vice versa. An example of such a change is illustrated here, with the referent in italics:}

Table 2

Mean Comprehension Latencies for Target Sentences With Referents in Clause B of Four Types of Context Paragraphs

\begin{tabular}{ccc}
$\begin{array}{c}\text { Type of Context } \\
\text { Paragraph }\end{array}$ & Latencies & Means \\
\hline 1. sA, B. C. & 1710 & 1701 \\
2. A sB. C. & 1692 & \\
3. A. sB, C. & 1661 & 1659 \\
4. A. B sC. & 1656 & \\
\hline
\end{tabular}

Type 3 (Context) Curious spectators lined the riverbank to watch the execution. While two sentinels stood at attention, workmen built a temporary gallows on the bridge. (Target) The sentinels were carrying loaded rifles. Type 4 (Context) Curious spectators lined the riverbank to watch the execution. Workmen built a temporary gallows on the bridge while two sentinels stood at attention. (Target) The sentinels were carrying loaded rifles. So each target sentence was preceded in one list by the referent in context Clause $B$ and in the other list by the referent in context Clause $C$. The mean number of words in Clauses $A$, $\mathrm{B}, \mathrm{C}$, and the target sentence were 11.4 (range 6-17), 10.7 (range 5-18), 10.7 (range 5-18), and 6.7 (range 5-9), respectively.

In other respects, Experiment 3 was identical to Experiment 2. There were 24 subjects, half men and half women. One subject was replaced for not following instructions. In the three-step analysis of the latencies, we used a 3.0-SD cutoff and had to replace only $3.0 \%$ of the scores.

\section{Results}

The mean latencies for the four conditions of Experiment 3 are shown in Table 3 . Subjects were $119 \mathrm{msec}$ faster on the average when the referent was in Clause $C$ than when it was in Clause B, and this was a highly reliable difference $\left[\min \mathrm{F}^{\prime}(1,46)=10.92, \mathrm{p}<.005\right]$. Subjects were only $12 \mathrm{msec}$ faster when the referent was in the main clause than when it was in the subordinate clause, which is obviously not a reliable difference. Nor was there a reliable interaction between the type and position of clause. In short, there was but one reliable finding: Referents were easier to retrieve when mentioned in Clause $\mathrm{C}$ than when mentioned in Clause B.

\section{GENERAL DISCUSSION}

In most circumstances, we do not feel we have understood a sentence until we have identified the objects, events, and states referred to by the definite

Table 3

Mean Comprehension Latencies for Target Sentences With Referents in One of Two Context Clauses

\begin{tabular}{lcc} 
Type of Clause & \multicolumn{2}{c}{$\begin{array}{c}\text { Context Clause Mentioning } \\
\text { Referent }\end{array}$} \\
\cline { 2 - 3 } Containing & B & C \\
\hline Main & 1663 & 1525 \\
Suborent & 1633 & 1532 \\
Means & 1648 & 1529 \\
\hline
\end{tabular}


noun phrases it contains. It is not enough to know that the speaker had referents in mind when he used the noun phrases. We must know for ourselves what those referents were. Identifying a referent entails, minimally, finding an entity about which we know other facts. For the noun phrase "the woman," as an example, finding the referent may mean determining that she is Mary, a person we have known for years and about whom we know a lot. Or it may mean realizing that she is the person the speaker introduced in the last sentence and about whom we know just one fact-the one asserted in the last sentence. In this study, we have shown that identifying a referent depends in part on where in the prior discourse it was mentioned last.

Experiments 1, 2, and 3 appear to demonstrate that the clause one back from the current sentence is a privileged place for the mention of referents. Referents mentioned in that clause are easy to pick out, whereas referents mentioned further back are relatively hard to pick out. The reasoning goes as follows. Subjects in Experiment 1 identified referents quickly when they were mentioned one sentence back, but comparatively slowly when they were mentioned two or three sentences back. But since the sentences in Experiment 1 each consisted of one clause, it was impossible to distinguish whether it was the last sentence or the last clause that had the privileged status. In Experiment 2, where every referent was mentioned two clauses back, it made little difference whether those clauses belonged to the first or to the second sentence back. The sentence boundary per se did not appear to be very important. In Experiment 3, where referents were either one or two clauses back and always within the last sentence, subjects were faster in identifying referents mentioned in the last clause than those mentioned two clauses back. The boundary between the first and second clauses back had direct consequences in the search for referents.

This conclusion, however, should be viewed with caution, as a comparison of Experiments 1 and 3 reveals. In Experiment 1, the discontinuity between one and two clauses back was a sizable $325 \mathrm{msec}$, whereas in Experiment 3, it was only one-third that size, $120 \mathrm{msec}$. Why the large discrepancy? For one thing, the last clause boundary in Experiment 1 was also a sentence boundary, which may have led to a larger effect. And the last sentence in Experiment 1 was slightly longer (12.1 words to 10.7 words) than the last clause in Experiment 3 ; the slightly greater content may have resulted in a more complete loss of information from the sentences preceding it. These factors-clause boundaries, sentence boundaries, and sheer amount of informational content-may work jointly to compound the effects of clause boundaries on the availability of entities mentioned in discourse. So, while the discontinuity in Experiment 1 may be real enough, its size is probably determined by multiple aspects of that experiment.
On reflection, this conclusion should not be surprising, for, as noted in the introduction, the privileged status of the last clause is just one of many factors that affect the availability of referents. The other factors that were mentioned-the grammatical roles of the noun phrase and its antecedent in their respective clauses, and the topic, scene, and assumed facts-also play significant roles. In the present experiments, we tried to hold these factors constant, but, except for the grammatical roles, that may have been impossible to do. To subordinate Clause $B$ to Clause $C$, or vice versa, is to explicitly point out to the reader that these two clauses are thematically related, that they bear on the same topic, belong to the same scene, and assume the same background facts. Indeed, compared to the independent clauses in Experiment 1, such subordination in Experiment 3 reduced the effect of the clause discontinuity by about two-thirds. Thus, thematic effects may be inherently confounded with clause vs. sentence boundary effects. What we have shown here is that at least the clause and sentence boundary effects are real.

One final note: subjects were often well aware of the relative unavailability of referents in clauses prior to the last one. In Experiment 1, they made two types of comments. First, they noted how difficult it was to identify a referent that was not mentioned in the last sentence. And their few failures to respond, which were counted as errors, occurred only when a referent was mentioned two or three sentences back. Second, subjects remarked on the relative awkwardness of these same sequences. The author of the sequence should not have returned to talk about something that was mentioned so long before. This violates the principles of good writing. These comments are just the ones that should be expected if the last clause has the privileged status it does. Speakers should refer to entities that are, as Chafe (1974) says, "on stage," and not to ones that have to be sought out in the wings.

\section{REFERENCE NOTE}

1. Charniak, E. Toward a model of children's story comprehension (M.I.T. A.I. Memo TR-266). Cambridge, Mass: Massachusetts Institute of Technology, 1972.

\section{REFERENCES}

Caramazza, A., Grober, E., Garvey, C., \& Yates, J. Comprehension of anaphoric pronouns. Journal of Verbal Learning and Verbal Behavior, 1977, 16, 601-610.

CARPENTER, P. A., \& Just, M. A. Integrative processes in comprehension. In D. LaBerge \& S. J. Samuels (Eds.), Basic processes in reading: Perception and comprehension. Hillsdale, N.J: Lawrence Erlbaum, 1978.

Chafe, W. L. Language and consciousness. Language, 1974, 50, 111-133.

Clark, H. H. The language-as-fixed-effect fallacy: A critique of language statistics in psychological research. Journal of Verbal Learning and Verbal Behavior, 1973, 12, 335-359. 
Clark, H. H. Inferences in comprehension. In D. LaBerge \& S. J. Samuels (Eds.), Basic processes in reading: Perception and comprehension. Hillsdale, N.Y: Lawrence Erlbaum, 1977.

CLARK, H. H. Inferring what is meant. In W. J. M. Levelt \& G. B. Flores d'Arcais (Eds.), Studies in the perception of language. London: Wiley, 1978.

Clark, H. H., \& Haviland, S. E. Psychological processes as linguistic explanation. In D. Cohen (Ed.). Explaining linguistic phenomena. Washington, D.C: Hemisphere, 1974.

Clark, H. H., \& Haviland, S. E. Comprehension and the givennew contract. In R. Freedle (Ed.), Discourse production and comprehension. Hillsdale, N.J: Lawrence Erlbaum, 1977.

Garvey, C., Caramazza, A., \& Yates, J. Factors influencing assignment of pronoun antecedents. Cognition, 1976, 3, 227-243.

Grice, H. P. Logic and conversation. In P. Cole \& J. L. Morgan (Eds.), Syntax and semantics. Vol. 3: Speech Acts. New York: Seminar Press, 1975.

Haviland, S. E., \& Clark, H. H. What's new? Acquiring new information as a process in comprehension. Journal of Verbal Learning and Verbal Behavior, 1974, 13, 512-521.

Hupet, M., \& LeBouedec, B. The given-new contract and the constructive aspect of memory for ideas. Journal of Verbal Learning and Verbal Behavior, 1977, 16. 69-76.

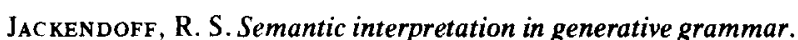
Cambridge, Mass: M.I.T. Press, 1972.

JARVELLA, R. J. Syntactic processing of connected speech. Journal of Verbal Learning and Verbal Behavior, 1971, 10, 409-416.

LANGACKER, R. On pronominalization and the chain of command. In D. Reibel and S. Schane (Eds.), Modern studies in English. Englewood Cliffs, N.J: Prentice-Hall, 1969.

LESS, R. B., \& KLIma, E. S. Rules for English pronominalization. Language, 1963, 39, 17-28.

Postal, P. Crossover phenomena. New York: Holt, Rinehart, \& Winston, 1971.

Ross, J. R. On the cyclic nature of English pronominalization. In To honor Roman Jakobson. The Hague: Mouton, 1967.

SPRINGSTon, F. J. Some cognitive aspects of presupposed corefer ential anaphora. Unpublished doctoral dissertation, Stanford University, 1975.

WINER, B. J. Statistical principles in experimental design. New York: McGraw-Hill, 1971.

YEKOVICH, F. R., \& W WLKER, C. H. Identifying and using referents in sentence comprehension. Journal of Verbal Learning and Verbal Behavior, 1978, 17, 265-277.

(Accepted for publication November 14, 1978.) 